\title{
Customizing and Curating e-Content with VitalStream
}

\author{
Viraj Kumar ${ }^{1}$,Sateesh Bhat ${ }^{2}$ \\ ${ }^{1}$ Department of Computer Science Engineering, PES University, Bangalore \\ ${ }^{2}$ Senior Product Manager, TeleVital India Pvt. Ltd., Bangalore \\ ${ }^{1}$ viraj.kumar@pes.edu \\ ${ }^{2}$ sateesh@televital.com
}

\begin{abstract}
A wealth of high-quality educational e-content has been developed over the past decade, and much of it is freely available. Instructors wishing to blend such content into their courses often find it useful to provide students with supplementary materials that (1) clarify the context in which the new content fits their particular curriculum, and (2) explainthe content, especially when the language or the accent of the instructor is unfamiliar for students. The latter point is especially important in a country as linguistically diverse as India, where instructors regularly use regional languages or Hindi to communicatemore effectively with students.In this paper, we describe an ongoing partnership to enhance a software productfor customizing and curating e-content developed by a company, based on requirements identified by an academic institution. We present examples where the institution's specific needs were first generalized(to address a wider category of similar concerns that other institutions may face), before being crystallized as new features in the software.Finally, we describe collaborative efforts to enhance the software by simplifying the access mechanism to e-content for students.
\end{abstract}

Keywords: e-content, customization, curation, blended learning.

\section{INTRODUCTION}

Ever since the "tipping point" in the early 2000's, the vast majority of the world's data has migrated from analogue to digital format (Hilbertand López, 2011). Educational data is also part of this rapid shift. Thistransformation opens up new avenues for academia-industry collaboration that are focused onmanaging this transition smoothly.In this paper we describean example ofsuch a collaborative effort between an academic institution and a software company, which has resulted in breakthroughs in customizing and curating e-content.

The internet has made it possible to disseminate highquality educational materials to a wider body of students than ever before. Over the past decade, several countries have launched efforts to help students access such content (Bassi, 2011). There is, however, a growing consensus that mere access is not enough - it is also necessary for all stakeholders (including instructors and students) to see the relevance of content to their curriculum.A clear example of this need is the usage of content created under India's National Programme on Technology Enhanced Learning (NPTEL).In an effort to minimize the inevitablemismatch between the objectives of e-content creators and end-users, NPTEL has attempted to standardize their course contentsby following the model curriculum of the All India Council for Technical Education (NPTEL, 2015).Even so, these resources are severely under-utilized because" material created under NPTEL does not conform to the syllabi of theuniversities", and hence"colleges are hesitant to use it" (Singh, 2012).

In order to address the significant challenge of matching econtent to the varied needs of institutions and students, we leverage the knowledge of the individuals who are ideally placed to understand these mismatches: instructors. In this paper, we describe a software tool that enables instructors to curate e-content and tweak it to fit the requirements of their institutions and students. This paper is organized as follows. Section2 places our contributions in relation to prior research. In Section 3, we describe how our tool has been enhanced by a collaborative effort between industry and academia. We illustrate how these enhancements have
PES University, Bangalore

viraj.kumar@pes.edu 
led to breakthroughs in search for relevant e-content in Section 4. Finally, in Section 5we present our conclusions, and discussdirections for futureimprovements to this tool as we continue our fruitful collaboration.

\section{RELATED WORK}

One answer to the problem of alignment is the use of blended learning techniques, where e-content is combined with "established classroom forms that require the physical co-presence of teacher and students" (Friesen, 2012). The assumption here is that,in addition to answeringquestions posed by students,instructors are in the best position to determine how the e-content should be contextualized andmodified (if necessary)to suit the needs of their students and institutions.Several easy-to-use software tools (both commercial and open-source) are now available to edit slides and video lectures, but modifying source content can raise copyright issues. Although NPTEL (and a few other educational e-content providers)distribute content under licenses that permit modifications (NPTEL 2015), it is possible for instructors to embellish videos without altering the underlying contentusing micro-notes(Kumar, 2014).

A micro-note is a digital footnote associated with a specific time-point within a video lecture, and is displayed at that point during video playback. In a blended environment, instructors often make interjections to clarifyan ambiguity, to point out an error, or to pose questions of their own. All such meta-content can be made available to students as micro-notes, and an extension of the open-source Moodle Learning Management System (LMS) supports the creation of simple (textual) micro-notes (Rajesh et al., 2015). In this work, we support a richer class of micro-notes that includes multiple-choice questions (MCQs). Additionally, faculty in a country as linguistically diverse as India may wish tocreate meta-contentin aregional language (or Hindi) that students are fluent in, even if the original e-content is in English.Our software supports this functionality as well. A schematic representation is shown in Fig. 1.

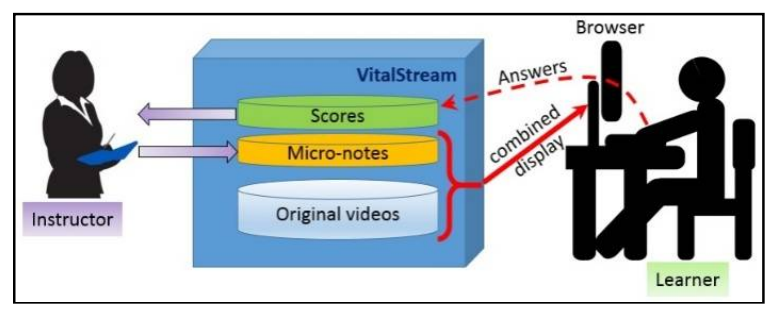

Fig. 1A schematic representation of our software tool (VitalStream)

It is important to note the difference betweenmicro-notes and other types of video annotations. YouTube Annotations and similar tools allow instructors to create annotations foreducational videoseasily. These tools are designed to help instructors collaboratively converge to a definitive set of meta-content for a given lecture, similar to the manner in which various contributors to a Wikipedia article aim for a consensus. Several studies have confirmed that learners benefit from this kind of meta-content (Cross et al., 2014; LaiolaGuimarães et al., 2010; Müller et al., 2010; Zimmermann and Weber, 2012).As we have noted, there may not always be a consensus on suitable meta-content for a certain lecture. Thus, micro-notesserve a complementary purpose: theyallow instructors freedom todiverge and to create meta-content that is customized to the specific needs of their students and institutions. It is, of course, possible that several instructors may find the content created by one instructor useful. At present, our tool only supports sharing meta-content within a particular institution, but websites such as TeachersPayTeachers.com do allow e-content to be shared more widely.

\section{From Curation to Customization}

VitalStream is a well-established software system that helps institutions make educational content available to students. There are two options for hosting content:(1) on the cloud, which requires students to have reliable accessto internet, but allows institutions to rent expensive equipment (instead of purchasing it outright); or (2) on institutional servers, where students can access content over the local network whenever they are on campus. At the partner institution for this research, we use the latter model. The videos we host are selected by our faculty from online repositories (e.g., NPTEL, Khan Academy, and Microsoft Research's portal for Massively Empowered Classrooms (MEC))for their compatibility with our curriculum (see Fig. 2).

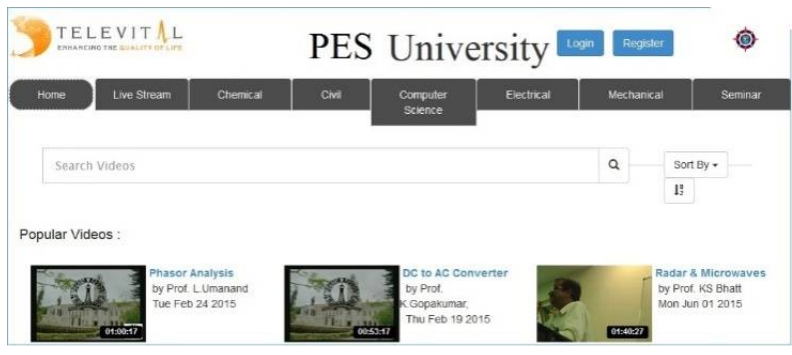

Fig. 2VitalStream software deployment at PES University

At present, only instructors can upload content, which is defined as videos and other supplementary content (slides, notes, etc.) associated with these videos.Instructors can also supply meta-data, as shown in Fig. 3, to helpstudents locate relevant content using the search bar.

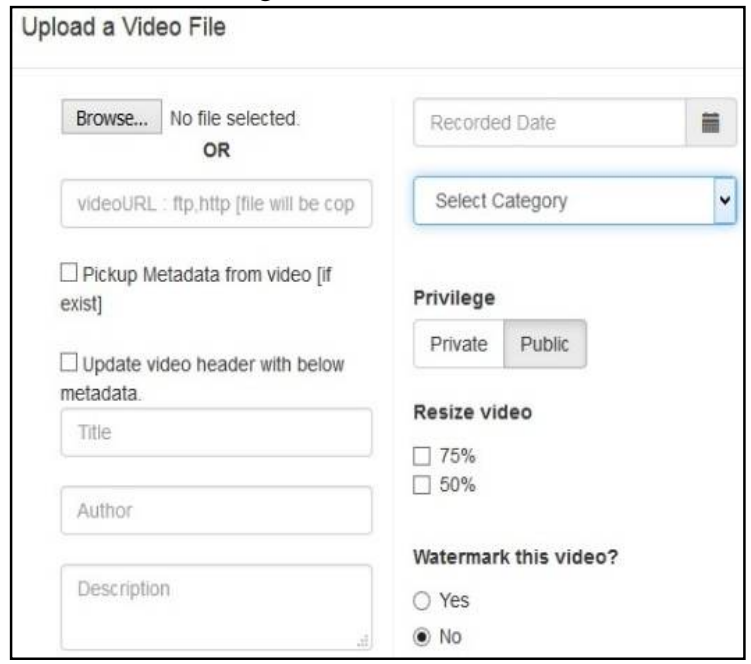

Fig. 3Options for tagging available to instructors when uploading videos 
Our industry-academic partnership began when instructors started blending these videos into their classes: they often found themselves wanting to pause a video at certain points and switch over to a related document, before resuming the video.We realized that thein-class experience for students and instructors would be greatly improved if this process was automated. We have implemented this functionality as shown in Fig. 4 (a) and (b), which shows a MCQ created by one of the authors for a Khan Academy video.

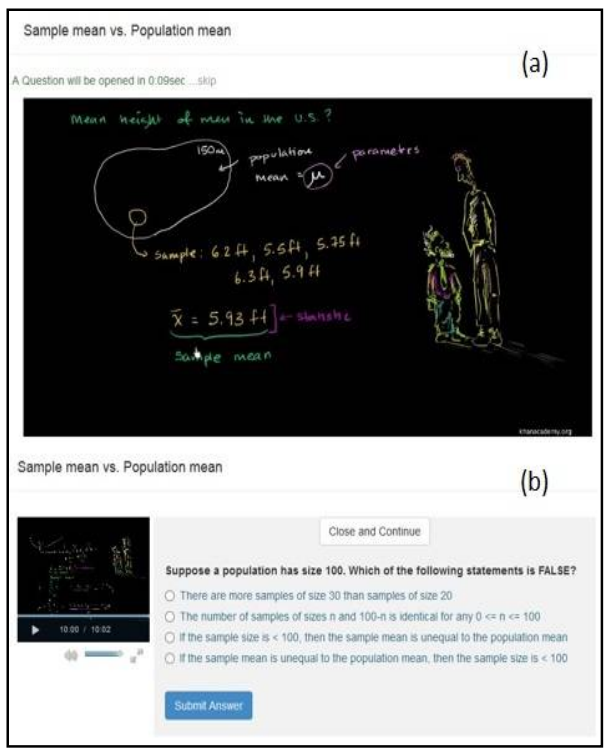

Fig. 4 A count-down indicates that the video will be paused (a), and then the MCQ is shown (b). Playback resumes on clicking "Close and Continue"

The manner in which students watch educational videos outside class can vary from one viewing to the next (Kim et al., 2014). For instance, a student may wish to assimilate the whole lecture before attempting the MCQ shown in Fig. 4(b).We therefore warn the viewer whenever the video is about to be paused, and allow the user to skip this material (Fig. 4(a)).

As we created these features, we recognized the value of our industry-academic partnership in enhancing the overall product: the academic community can test the software in the field andsuggest areas of improvementbased on their own experience and familiarity with relevant research, and the industry partner can use their wealth of experience to design elegant and easy-to-use interfaces to implement the desired features. An example of the latter is the intuitive interface for creating micro-notes. The instructor pauses the video at the desired time-point, and the tool automatically creates a "hook" onto which additional materials can be tethered. Fig. 5 shows how the instructor can associate a MCQ, for example. The instructor can also specify whether the student must be logged into their own account to view the question or not. In the former case, the student's answer can be recorded and be used for evaluation purposes.

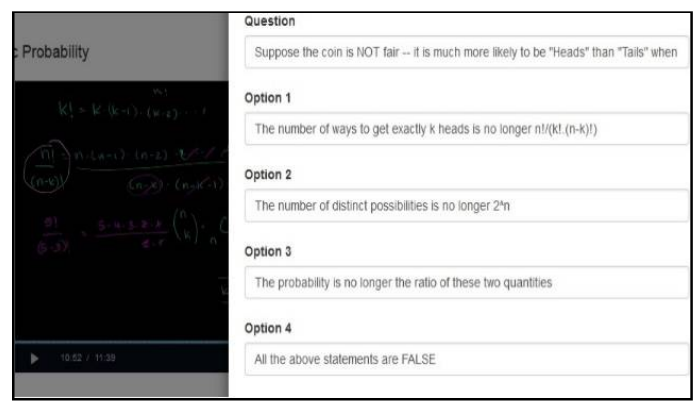

Fig. 5Interface for creating a multiple-choice question (MCQ) as a micronote.

\section{Advanced Content Search}

As the size of an institution's corpus of educational content grows, it becomes imperative to help users locate relevant e-content quickly. In many cases, videos are untagged, or are extremely large and have no internal tags to identify specific segments. For instance, NPTEL videos are often 60 minutes long, and efforts are underway to create tables of content in a semi-automated way.It is possible to extract appropriate keywords automatically from audio and video streams (Biswas et al., 2015; Gandhi et al., 2015), but the process is error-prone and computationally expensive. As a result, NPTEL has devoted significant resources towards (manually) transcribing thousands of hours of lectures (http://textofvideo.nptel.iitm.ac.in). Although this resource allows students to watch videos with subtitles, it can also assist powerful searches over videos. For instance, a tool has been developed to find relevant NPTEL videosegments to answer questions from the Graduate Aptitude Test in Engineering (Mohan et al., 2015).

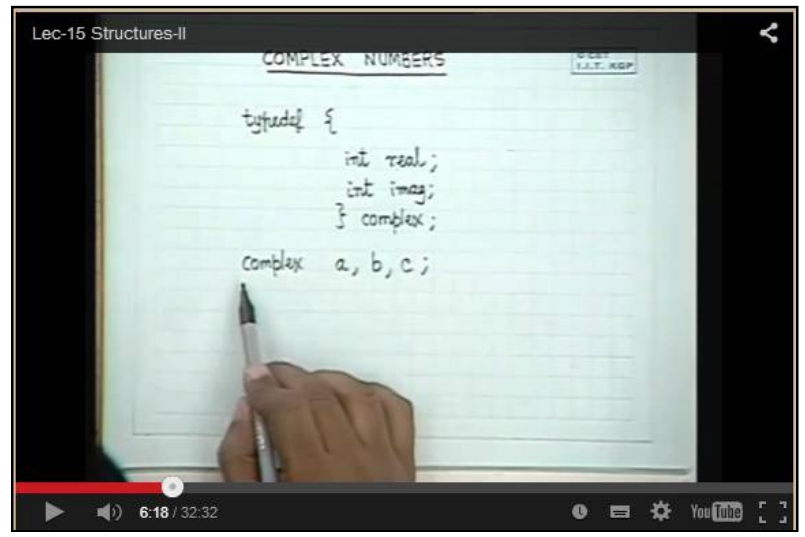

Fig. 6An NPTEL video explaining the concept of a "struct" in the C programming language, using complex numbers as an example.

In a similar spirit, we have extended VitalStream'ssearch capabilities to answer queries based not only on tags, but also by scanning the contents of micro-notes. The result is an ability to perform new kinds of searches over videos. To understand this capability, consider the following example.

Recall that micro-notes can be used in many ways, and are not restricted to any particular language. Fig. 6 shows a segment of an NPTEL video from our corpus where one of ourinstructorshas created two micro-notes. The first is in 
English, and points out a minor syntax error in the code fragment. (NPTELvideos are carefully reviewed, but the fact that the keyword "struct" is missingin this segment has escaped detection). The second micro-note is meant for students who may have studied complex numbers in Hindi at school, and therefore refer to them as "सम्मिश्रसंख्या" (transliterated as sammishrasankhya in the micro-note).

Suppose an instructor blends this video into a lecture on $\mathrm{C}$ programming, pointing out the Hindi terminology and the syntax error. Now consider two students who are trying to locate this particular video segment several weeks later. The first student only remembers that it had "something to do with sammishrasankhya", and the second only recalls that there was "somekind of error in the code". To the best of our knowledge, no existing techniques can help either of these students, because there is nothing in the video to match queries such as "sammishrasankhya" or "error in the code". Since this meta-content was created by the instructor and recorded as micro-notes, VitalStreamcan immediately locate this segment in our corpus. As an institution's collection of micro-notes associated with videos grows, the tool's abilityto perform such advanced searches increases.

\section{Conclusions And Future Work}

As mentioned in Section 3, instructorsand students access e-content over the local network on our campus using any popular browser. Our immediate goal is to recreate this browser-based experience of videos and micro-notes in an offline manner, especially for students who do not have access to internet at home.For micro-notes that are purely textual, this is immediately possible: all such content can be downloaded as a single SubRipText (SRT) file, together with the video file, and can be played on most computers (and even on certain televisions) as subtitles. Note that the viewer can no longer selectively skip content.However, certain kinds of micro-notes, e.g., multiple-choice questions, cannot be meaningfully reduced to video subtitles.

The proliferation of smartphones presents a viable solution to this problem. Many students can now afford at least a basic smartphone, especially one that is purchased secondhand (Rangaswamy and Cutrell, 2012). Here, we again see an excellent opportunity for strengthening our partnership between industry and academia. Most of our undergraduate students are keen to acquire app development skills, after which they are anxious to hone their talents on meaningful projects. Our plan is to hire committed students as interns to prototype the development of mobile apps for displaying videos with textual and interactive micro-notes.

The mobile platform offers several exciting possibilities, which we are also keen to explore. To begin with, although our current tool does not allow students to create micronotes, such a feature makes sense in an app for students. Such micro-notes can be shared over social media and other channels, thereby digitizing one of the final frontiers of analogue data in education:hand-written (or photocopied) notes.

It is also possible to utilize mobile devices in a blended environment. At present, the instructor typically projects a video, and all students must follow along at the same pace. Watching such a video on a personal device allows students to pause, rewind and skip ahead to suit their own pace. The problem with poor acoustics in the classroom, particularly on warm days with ceiling fans humming, can also be alleviated when students use personal earpieces. We are presently investigating the challenges of conducting classes in such a manner, particularly with ensuring that students are actually engaged in scholarly activities instead of other distractions on their mobile devices. The synergistic nature of our partnership permits us to conduct such experiments.

Finally, there are numerous ways in which we can improve the interoperability between VitalStream and other learning technologies. For instance, we have stated in Section 3 that VitalStream can evaluate student answers to questions embedded as micro-notes within videos. If these scores are to be used for evaluation, our software needs to be able to export (and import) data in a format compatible with APIs for Learning Management Systems such as SCORM and xAPI. As we work towards these goals, we are constantly engaged in improving and testing our software in a realistic environment.

\section{ACKNOWLEDGEMENTS}

We would like to thank the respective heads of our partner institutions, the Vice Chancellor of PES University, Dr.K.N.B. Murthy and the CEO of VitalStream, Mr. Kishore Kumar Rao. We would also like to thank all instructors and researchers who have helped us to compile a rich set of micro-notes, and have given us their valuable suggestions for improving the software.

\section{REFERENCES}

Bassi, R. (2011) ICTs in Education (ICT4E) Policies and Plans worldwide. URL: http://www.gesci.org/assets/files/Knowledge\%20Centre/co untry-policies.pdf (last accessed on September 29, 2015)

Biswas, A., Gandhi, A. and Deshmukh, O. (2015) MMToC: A MultimodalMethod for Table of Content Creation in Educational Videos, Proc. of the $23^{\text {rd }} \mathrm{ACM}$ International Conference on Multimedia

Cross, A., Bayyapunedi, M., Ravindran, D., Cutrell, E., and Thies, W. (2014) VidWiki: Enabling the Crowd to Improve the Legibility of Online Educational Videos, Proc. of the $1^{\text {st }}$ ACM Conference on Computer Supported Cooperative Work and Social Computing

Friesen, N. (2012) Report: Defining Blended Learning, URL:

http://learningspaces.org/papers/Defining_Blended_Learnin g_NF.pdf (last accessed on September 29, 2015)

Gandhi, A., Biswas, A. and Deshmukh,O. (2015) Topic Transition inEducational Videos Using Visually Salient Words, Proc. of the $8^{\text {th }}$ InternationalConference on Educational Data Mining 
Hilbert, M. and López, P. (2011) The World's Technological Capacity to Store, Communicate, and Compute Information, Science,vol. 332, no. 60, pp. 60-65, doi: $10.1126 /$ science. 1200970

Kim, J., Guo, P. J., Seaton, D. T.,Mitros, P.,Gajos, K. Z. and MillerR. C. (2014) Understanding in-video dropouts and interaction peaksin online lecture videos, Proc. of the $1^{\text {st } A C M}$ conference on Learning @ Scale.

Kumar, V. (2014) Enhancing Video Lectures with Digital Footnotes, Proc. of the $44^{\text {th }}$ IEEE Conference on Frontiers in Education, pp. 1-3, doi: 10.1109/FIE.2014.7044212.

LaiolaGuimarães, R., Cesar, P., and Bulterman, D. (2010) Creating and sharing personalized time-based annotations of videos on the web, Proc. of the $10^{\text {th }}$ ACM Symposium on Document Engineering

Mohan, K., Desai, A. R., Ashish, M.P., and Kumar, V. (2015) Enhancing Digital Educational Repositories by Linking Videos and Examinations, in Proc. of the $7^{\text {th }}$ International IEEE Conference on Technology for Education (to appear)

Müller, S., Miller, G. and Fels, S. (2010) Using temporal video annotation as a navigational aid for video browsing, Proc. of the $23^{\text {rd }}$ ACM Symposium on User Interface Software and Technology

NPTEL. (2015) Frequently Asked Questions. URL: http://nptel.ac.in/faq.php (last accessed on September 29, 2015)

Rajesh, K., Goudar, R. and Kumar, V. (2015) Enabling Micro-notes in Moodle for Educational Videos, in Proc. of the $7^{\text {th }}$ International IEEE Conference on Technology for Education (to appear)

Rangaswamy, N. and Cutrell, E. (2012) Anthropology, development andICTs: Slums, youth and the mobile internet in urban India,International Conference on Information and CommunicationTechnologies and Development Special Issue, vol. 9, no. 2, pp. 51-63

Singh, A. K. (2012) Minutes of the 23rd Meeting of Project Approval Board of National Mission on Education through Information and Communication Technology. URL: http://www.nmeict.ac.in/officeDocumentUploaded/19-52014/2012-02-28_Meeting23.pdf (last accessed on September 29, 2015)

Zimmermann, T. and Weber, M. (2012) CoVidA: penbased collaborative video annotation, Proc. of the

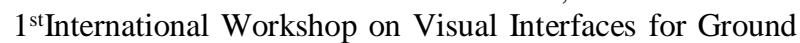
Truth Collection in Computer Vision Applications 